\title{
Rita's 102!!
}

\section{Rita Levi-Montalcini • Richard A. Knight • Pierluigi Nicotera • Giuseppe Nisticó • Nicolas Bazan • Gerry Melino}

Published online: 16 March 2011

(C) Springer Science+Business Media, LLC 2011

It is an honour for us to write this Editorial introducing a set of reviews in celebration of Rita Levi-Montalcini's 102nd birthday. Rita is the oldest living Nobel Laureate and the first ever to live for more than a century. Without her identification of nerve growth factor (NGF) as a neuronal survival factor, well before many other endogenous cell survival ligands were identified, academic neurobiology would not be on the translational threshold that it stands today. Indeed, the consensus that NGF may well revolutionise clinical therapy for the increasing public neurodegenerative burden is now at an advanced stage of optimisation of formulation and delivery. Rita, herself, has contributed significantly to this, partly by optimising largescale production of rhNGF, and by showing, in an

R. Levi-Montalcini · G. Nisticó · G. Melino European Brain Research Institute,

Rome, Italy

R. A. Knight $\cdot$ G. Melino

Medical Research Council Toxicology Unit,

Leicester, UK

\section{P. Nicotera}

Deutsches Zentrum für Neurodegenerative Erkrankungen (DZNE), Bonn, Germany

\section{N. Bazan}

LSU Neuroscience Center of Excellence,

New Orleans, LA, USA

\section{G. Melino $(\square)$}

Biochemistry Laboratory IDI-IRCCS, Department of

Experimental Medicine, University of Rome Tor Vergata, Rome, Italy

e-mail: melino@uniroma2.it

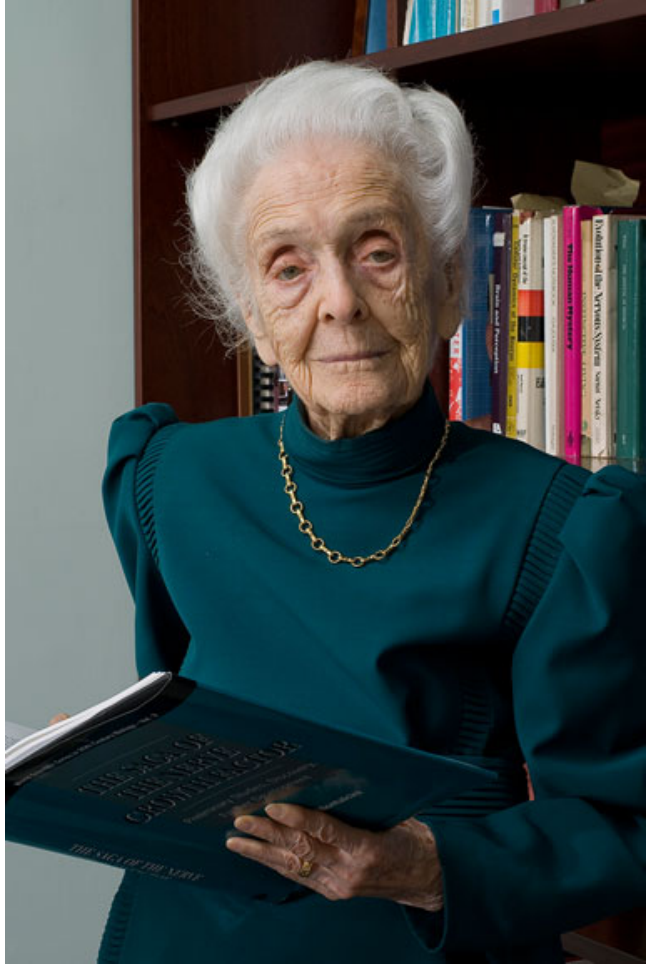

admittedly small-scale human study, that NGF therapy can reduce retinal ganglion cell loss in patients with glaucoma. In addition, it has been known for some time that inhibiting endogenous NGF production, as in the AD11 mouse, leads to neurodegeneration, which can be reversed by increasing endogenous NGF expression and by exogenous NGF.

As many of you know, Rita was born in Turin and graduated from the Medical School there in 1936. Forced from formal academic life by political events, she 
performed experiments on the growth of nerve fibres in chick embryos from a home laboratory-experiments which were to form the foundation of her later career. In 1946, she moved to Washington University in St Louis, where, in collaboration with Stanley Cohen in 1952, she found that mouse tumour cells release a factor which causes rapid growth of nerve cells. This was, of course, NGF, and Rita and Stanley Cohen shared the Nobel Prize in Physiology or Medicine for its discovery in 1986. Rita became a full Professor in St. Louis in 1958, but from 1962, divided her time between St. Louis and a newly established research unit in Rome. Subsequently, she became director firstly of the Research Centre for Neurobiology and then the Laboratory of Cell Biology in the CNR in Rome. To further develop potential therapeutics based on NGF, and to attract world class scientists to its cause, she set up the European Brain Research Institute (EBRI), also in Rome, in 2002. The work of EBRI covers a wide range of neurodegenerative pathologies, and we think it is appropriate that this broad spectrum is reflected in the content of the reviews published here [1-8].

Two reviews focus in particular on Parkinson's disease (PD). Miguel Martins' group review the data on the role of the mitochondrial serine protease, $\mathrm{HtrA} 2 / \mathrm{Omi}$, a protein which has also been implicated in amyotrophic lateral sclerosis, in the maintenance of mitochondrial quality control. Heterozygous missense mutations have been described in some cases of sporadic PD, and HtrA2 is downstream of PTEN-induced putative kinase 1 (PINK1), a protein also mutated in familial PD. Martins group speculate that the PINK1/HtrA2 pathway is important for maintaining the molecular quality of mitochondria (possibly through hsp70), and PINK1 independently, through its recruitment of PARKIN, also maintains organelle quality through mitophagy. In a second review, Tomasetti's group address the central question of why dopaminergic neurons of the substantia nigra are the target of degeneration in PD. They concentrate on the role of orthodenticle protein homologue 2 (Otx2), a protein abundant in precursors and show, using conditional Otx2 gain and loss of function mice, that there is an inverse transcriptional relationship between Otx2 and the glycosylated form of the dopamine transporter (glyco-Dat). Thus, neurons expressing high levels of glyco-Dat are vulnerable to degeneration, whereas cells expressing high levels of Otx2 (and therefore, low glyco-Dat) are resistant to the toxin, MPTP. Another inverse relationship, between the $\mathrm{NKCC} 1$ and $\mathrm{KCC} 2$ chloride transporters, appears to contribute to the excitatory role of GABAnergic interneurons in the late embryonic and early postnatal hippocampus. Thus, a higher relative expression of NKCC1 allows chloride influx resulting in synaptogenesis, and as $\mathrm{KCC} 2$ expression increases in later postnatal life, so GABA becomes inhibitory. Cherubini's review deals with the potential contribution of this system to neurodegeneration by showing, for example, that NKCC1 knockdown in vivo reduces synaptic transmission and decreases arborisation: indeed, there is some evidence that GABA may also become excitatory in neurodegeneration perhaps associated with reduced expression of $\mathrm{KCC} 2$.

Three of the reviews deal with more general aspects of neurodegeneration. Rodriguez and Verkhratsky give an overview of the role of neuroglia and suggest that the early loss of astroglia compromises synaptic maintenance with the failure of neurotransmission and cognitive decline. The gliosis seen in later stages is seen as a response to plaque deposition. The link between longevity and neurodegeneration is explored by Nicotera's group, who discuss the relationship between mTOR inhibition (induced, for example, by nutrient restriction), which leads to increased autophagy and lifespan extension, but also prevents amyloid-induced cognitive decline.

Finally, two reviews deal with the involvement of two proteins perhaps more familiar to the oncologist-PML and p73. In cancer, PML is a modulator of p53-mediated apoptosis and expression of PML is lost in many cancers. However, in conditions such as spinocerebellar ataxia (SCA), PML associates with Ataxins in nuclear aggregates and may contribute to the impaired proteasomal clearance of aggregated proteins in SCA and Huntington's. The phenotype of the p73 null mouse is neuronal with hippocampal dysgenesis and loss of the lower blade of the dentate gyrus. Expression of p73 increases with neuronal differentiation and p73 overexpression enhances neurite outgrowth, and there is now evidence that the p73 axis is dysregulated in Alzheimer's disease.

In publishing these reviews [1-8], we aim to provide the readers with comprehensive overviews of the current status of the science in these areas. In addition, we hope that they provide the stimulus for the making of new synaptic connections and thus, scientific advances, so necessary for the amelioration of the dramatic consequences caused by neurodegenerative disease.

\section{References}

1. Bano D, Agostini M, Melino G, Nicotera P (2011) Ageing, neuronal connectivity and brain disorders: an unsolved ripple effect. Mol Neurobiol 43:124-130

2. Cherubini E, Griguoli M, Safiulina V, Lagostena L (2011) The depolarizing action of GABA controls early network activity in the developing hippocampus. Mol Neurobiol 43:97-106

3. de Castro IP, Martins LM, Loh SH (2011) Mitochondrial quality control and Parkinson's disease: a pathway unfolds. Mol Neurobiol 43:80-86

4. Killick R, Niklison-Chirou M, Tomasini R, Bano D, Rufini A, Grespi F, Velletri T, Tucci P, Sayan BS, Conforti F, Gallagher E, Nicotera P, 
Mak TW, Melino G, Knight RA, Agostini M (2011) p73: a multifunctional protein in neurobiology. Mol Neurobiol 43:139-146

5. Rodríguez JJ, Verkhratsky A (2011) Neuroglial roots of neurodegenerative diseases? Mol Neurobiol 43:87-96

6. Salomoni P, Betts-Henderson J (2011) The role of PML in the nervous system. Mol Neurobiol 43:114-123
7. Simeone A, Di Salvio M, Di Giovannantonio LG, Acampora D, Omodei D, Tomasetti C (2011) The role of Otx2 in adult mesencephalicdiencephalic dopaminergic neurons. Mol Neurobiol 43:107-113

8. Stark D, Bazan N (2011) Neuroprotectin D1 induces neuronal survival and downregulation of amyloidogenic processing in Alzheimer's disease cellular models. Mol Neurobiol 43:131-138 